Thorax (1946), 1, 188.

\title{
THE EFFECT OF ASBESTOS, AND OF ASBESTOS AND ALUMINIUM, ON THE LUNGS OF RABBITS
}

BY

\author{
E. J. KING, J. W. CLEGG, AND V. M. RAE \\ From the British Postgraduate Medical School and the Brompton Hospital, London*
}

The purpose of these experiments was to test two questions: (a) whether shortfibre asbestos is less damaging in the lungs of the rabbit than is long-fibre asbestos, and (b) whether metallic aluminium will suppress any toxic effect produced by either the short- or long-fibre asbestos.

Early work with experimental asbestosis in animals has led Gardner, of Saranac Lake (Gardner, 1937, 1938, and 1940), to suggest a "mechanical" theory of asbestosis. In contrast to quartz and other forms of free silica, which appeared to become more toxic as their particle size decreased, the shorter the fibre of the asbestos dust used, the less tissue reaction was produced. In fact, asbestos dust which had been well ground in order to eliminate all long fibre produced almost no pathogenic effects in tissue at all. On the other hand, long-fibre asbestos, or the presence of long-fibre particles amongst dust of finer size, produced a diffuse fibrous response. The pathogenic effect with long-fibre asbestos, however, was obtained only in the lungs. In all other tissues asbestos of any length appeared to produce nothing other than a foreign-body reaction. These facts led Gardner to conclude that it was only in tissue where rhythmic movement takes place that asbestos can exert a pathogenic action, and then only if the particles are of sufficient size to produce mechanical irritation.

Gardner (1942) found that the dust of chrysotile asbestos produced markedly different pathogenic responses according to the animal used. The table reproduced from his paper summarizes his findings.

We considered using cats as experimental animals, but it was felt that they were too difficult to handle in any large number to make it possible to carry out the investigation on a sufficiently large scale. Rabbits were finally decided on, as, according to Gardner's results, they show a fair possibility of producing

* This work is a development of an investigation begun by the late Dr. T. H. Belt. 
Pulmonary Responses of Different Species to Chrysotile Asbestos

(GARDNER, 1942)

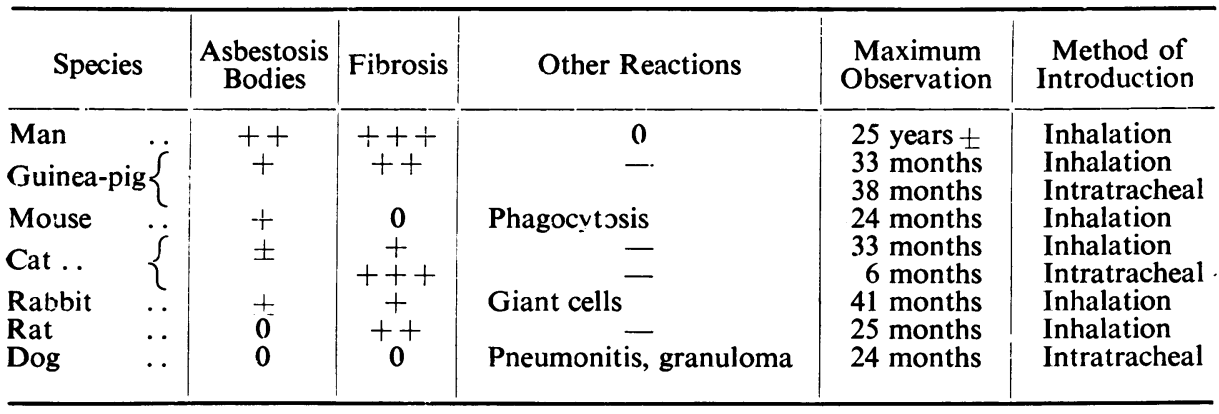

asbestos bodies, and do not appear to be much inferior to the rat for the production of fibrosis. Furthermore, the rabbit has the advantage that multiple administrations of the dust can be made without the necessity of operation. Whereas with the rat it is necessary to dissect open the neck and expose the trachea in order to carry out intratracheal insufflation, it is possible to introduce a rubber tube directly into the trachea of the rabbit by insertion through the mouth. This greatly facilitates administration, and administration of dust can be repeated as often as desired with no great trouble. In the experiments to be described, groups of rabbits have been treated with dust in this manner at monthly intervals and the animals sacrificed at varying times in order to observe the effect of different periods of exposure of the lungs to asbestos, and asbestos plus aluminium, and also the effect of few or multiple administrations.

\section{Methods}

The dusts used were Rhodesian asbestos cut into two standard lengths, $2.5 \mu$ and $15 \mu$, by a special microtome apparatus.* All samples used were kindly prepared for us by Mr. Dawson, of Turner Bros. Asbestos Co., Ltd.

The aluminium was a very fine powder consisting of particles of metal. It was prepared for us by Dr. Blaisdell of the Canadian Research Team who have been engaged on therapeutic trials with the treatment of silicosis by metallic aluminium dust, and is, in fact, the identical aluminium dust used in Canada for administration to humans (Crombie, Blaisdell, and MacPherson, 1944).

The rabbits were fully grown animals, healthy and of good stock, and weighed approximately $2 \frac{1}{2} \mathrm{~kg}$.

The asbestos dust samples were prepared for administration by suspension of the powder in physiological saline solution, $100 \mathrm{mg}$. of asbestos being contained in $4 \mathrm{ml}$. of saline. The mixture was sterilized by heat on a steam bath and cooled to body temperature. The metallic aluminium was mixed in the proportion of $2 \mathrm{mg}$. for each $100 \mathrm{mg}$. of asbestos.

* Asbestos is very brittle, and while the $2.5 \mu$ fibre asbestos was almost entirely composed of fibres of that length, it was not possible to produce powder of asbestos fibres all $15 \mu$ long, as fragmentation occurred during the cutting. Well over 80 per cent of the fibres, however, were close to $15 \mu$ in length. 
The administration was carried out as follows. A fine rubber catheter, of the type used for rabbits, was dipped in liquid paraffin. The rabbit, without any anaesthetic, was tied belly down on a board on the operating table, a pair of forceps inserted in the mouth behind the front teeth, the head drawn forward, and the mouth held open. The rubber tube was slipped down the throat and usually went straight into the trachea (with the animal on its back the tube will usually slip into the oesophagus). The tube was inserted for a length of about 3 in., when obstruction to its passage was felt. At this point the end of the tube was against the bifurcation of the bronchi. The presence of the tube in the trachea could be tested by holding a tuft of the animal's fur over the open end of the rubber tubing. The rhythmic movement of the fur to and from the tube, caused by inhalation and exhalation of air, was immediately seen if the tube was in the trachea. The flask containing the dust suspension was well shaken, and $4 \mathrm{ml}$. (100 mg. of the dust) drawn into a $5-\mathrm{ml}$. syringe (without needle). The teat of the syringe was inserted into the end of the rubber tube, the board on which the animal was tied was raised into a semi-vertical position, and the dust injected. The syringe was removed from the tube and filled with air, which was injected through the tube in order to wash in the remainder of the asbestos suspension. The animals usually coughed slightly, but did not seem to experience much discomfort. They were held in a vertical position for a minute or two to allow the mixture to drain into the lungs, and were then released.

These administrations of $100 \mathrm{mg}$. of asbestos, or of asbestos plus aluminium, were given to each rabbit once a month. The animals were maintained in large cages in an open courtyard during the summer months, and in small metal cages in a heated building during the winter. There were the usual deaths which inevitably occur in an animal population. The lungs of these animals, and those of others killed at intervals, were distended with formaldehyde-alcohol-saline mixture, and after fixation were cut up into blocks for histological section.

Five sections were made from each block, one each for haematoxylin and eosin staining, Van Gieson's connective tissue stain, and Wilder's reticulin stain. Two sections were mounted unstained and incinerated at $400^{\circ}$ to burn off the whole of the organic material. The mineral residue which was left helped to illustrate the inorganic "skeleton" of the lung tissue. The non-asbestos components of the inorganic residue were removed from the rest of the slide by soaking it in hydrochloric acid. All water and acid-soluble material was in this way removed, leaving the asbestos fibres behind in the position they occupied in the lung. It was thus possible to relate the mineral content of the lung to the structures seen in the other slides. Aluminium appeared only partially to be removed by the acid treatment of the incinerated section. Detailed histological examinations were carried out on all sections by both ordinary and polarizing microscopes.

\section{RESULTS}

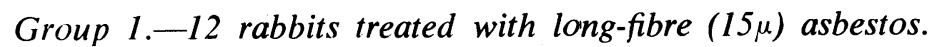

The survival time varied greatly; a few animals died between 2 and 3 months, others living as long as 19 months. On the whole the tissue reaction increases with the longer survival time, and is of two different types. The earlier is a foreign-body reaction composed of macrophages and foreign-body giant cells. This is nodular, usually occupies an area of 2-8 alveolar spaces and is associated with the presence of asbestos fibres, which appear as refractile bodies within the nodule (Figs. 1 and 2). 


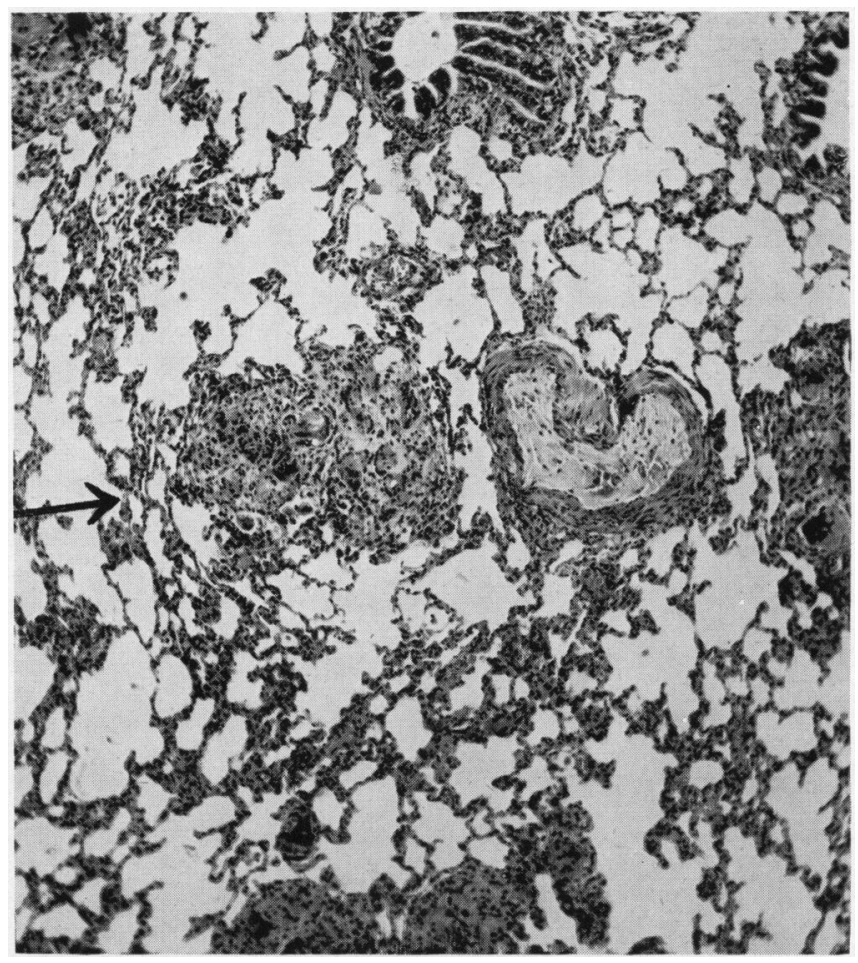

Fig. 1.-Long-fibre asbestos showing nodular distribution of foreign-body reaction $(\times 60)$.

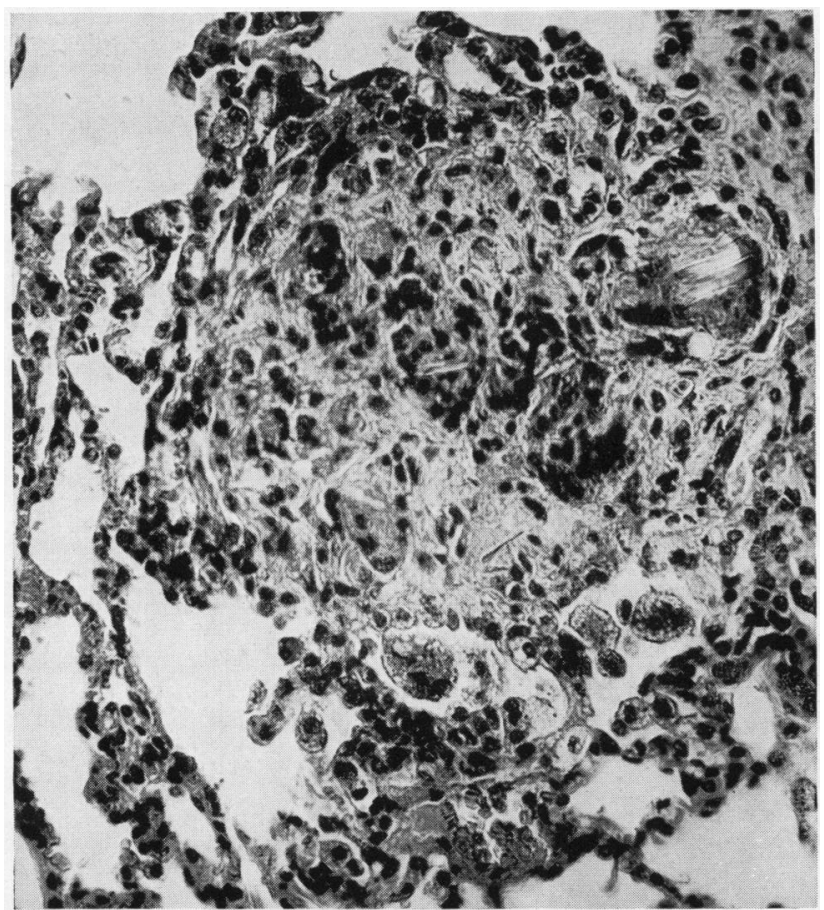

FIG. 2.-High-power view of nodule marked with arrow in Fig. 1, showing asbestos fibres in the nodule $(\times 240)$. 


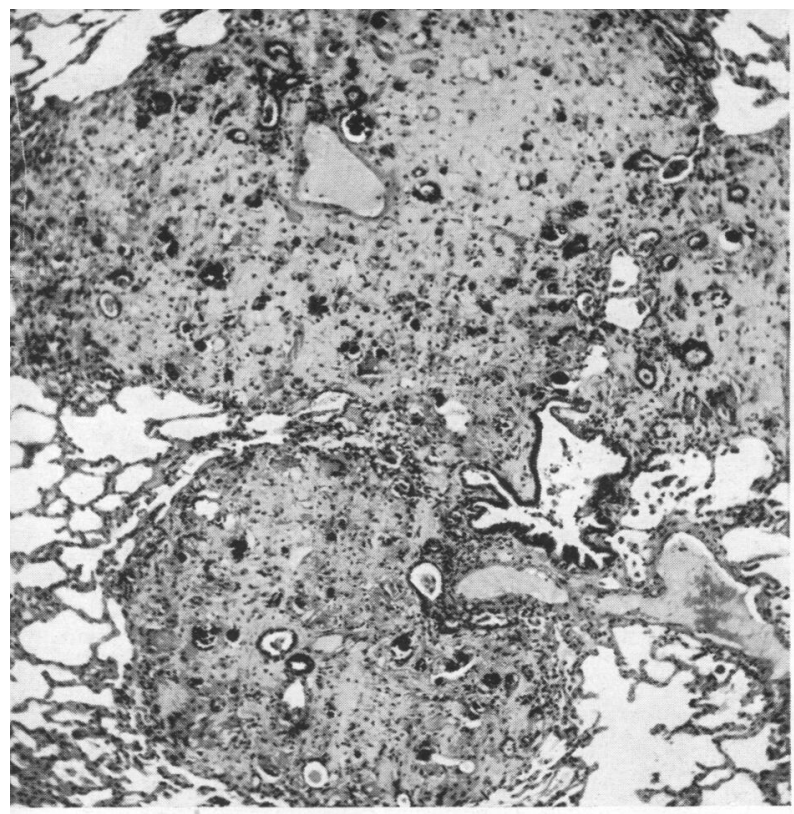

Fig. 6

FIGS. 6, 7 and 8.-Long-fibre asbestos and aluminium. Serial section showing black pigment in $\mathrm{H}$ and $\mathrm{E}$ section $(6)$; the pigment sti!l present in the incinerated section (7). Treatment of the incinerated section by washing in hydrochloric acid has removed the black pigment $(8)(\times 60)$.

FIG. 9.-Short-fibre asbestos. Hyperplastic lymph node showing phagocytes loaded with pigment $(\times 160)$.

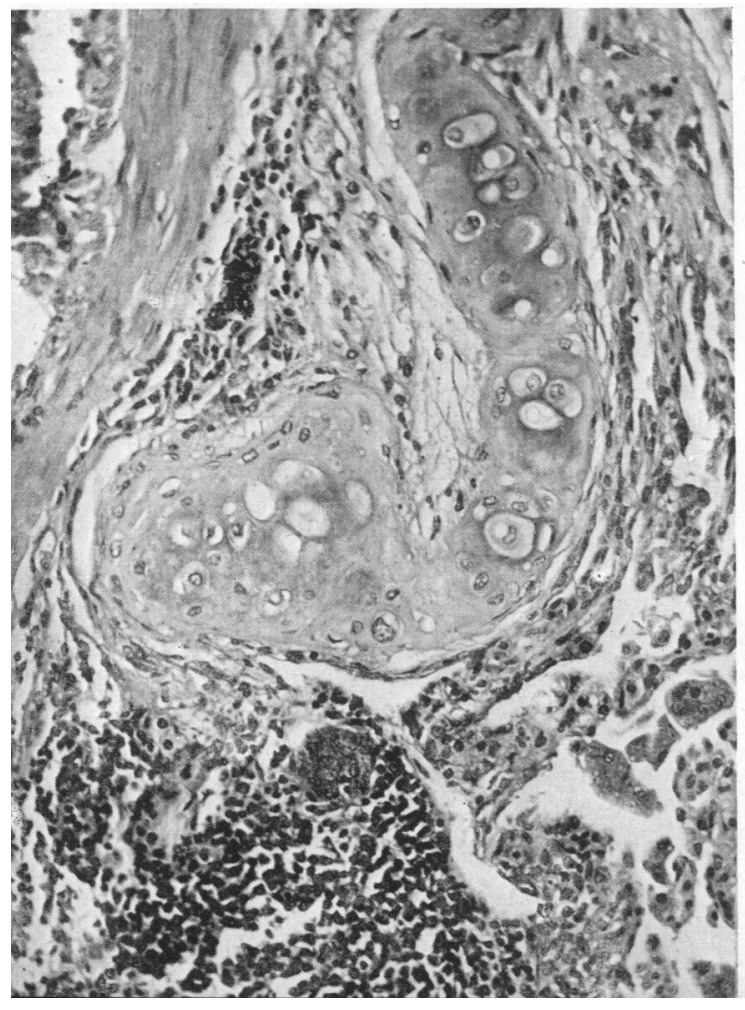

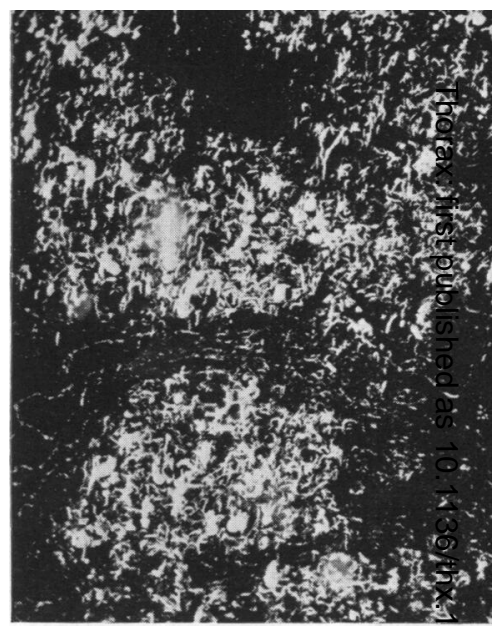

Fig. 7.

$\omega$

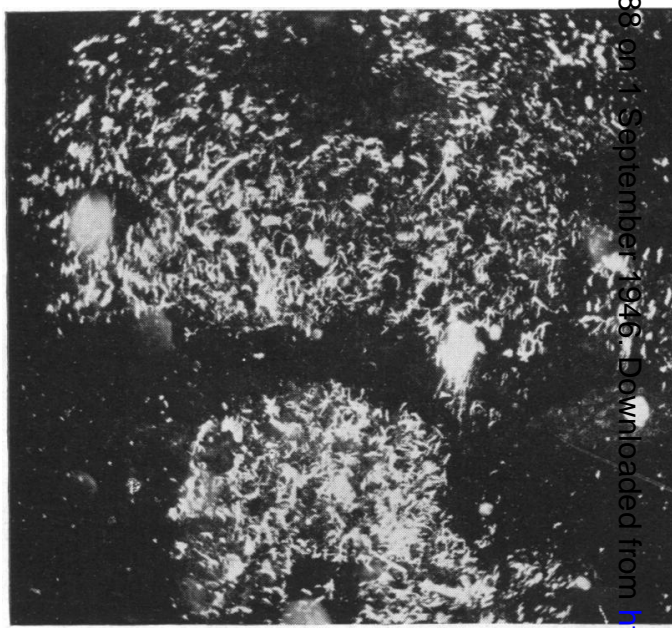

FIG. $\mathcal{E}$

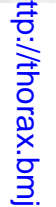

Fig. 10.-Short-fibre asbestos. Area of interstigial fibrosis and emphysema $(\times 28)$.

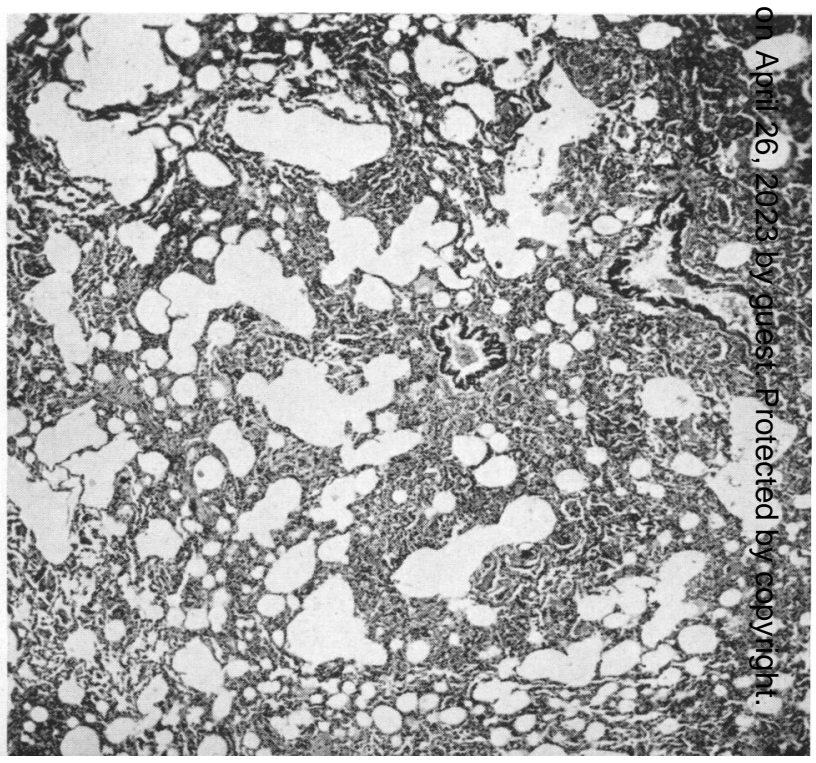


Overlapping the foreign-body giant-cell process, but generally occurring somewhat later, there is a more permanent reaction resulting in the fixation of asbestos in situ. The distribution is nodular. In the haematoxylin and eosin stained sections there are many nodules occupying from 2-8 alveolar spaces and composed of rather pale-staining, somewhat acellular areas of connective tissue. An increase in reticulin can be demonstrated by Wilder's method in these areas; and the presence of asbestos fibres, which are usually invisible in haematoxylin and eosin sections, can be demonstrated in the ashed section (Figs. 3, 4, and 5). These nodules are strictly comparable with, but less intense than, the nodules described by Belt (Belt and King, 1945) as a standard reaction to quartz (Brazilian pebble).

Dotted about these nodules there are deposits of two forms of pigment. There is a brown dust-like pigment which is frequently found in relation to the foreignbody giant-cell reaction. In the pale-staining connective tissue nodules there are much larger masses of pigment which stain densely with haematoxylin. The larger of the masses tend to be spherical, but the smaller masses are frequently rod-like. The frequency of this black pigment increases with the survival time of the animals. The black pigment is present in the ashed specimen, but is washed out by hydrochloric acid. The nature of this pigment is, at the present time, obscure (Figs. 6, 7, and 8).

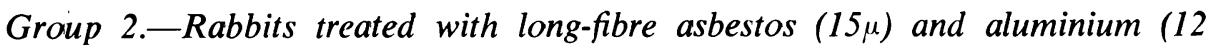
animals).

The number of animals and the survival time in this experiment are strictly comparable with Group 1. The general pattern of the tissue reaction in this experiment is similar to that found in Group 1. The chief difference lies in the overlapping of the foreign-body reaction and the reticulinosis. It seems that the addition of aluminium increases and prolongs the foreign-body giant-cell reaction without inducing any marked inhibition of the appearance of the reticulinosis. The same pigments are present in these experimental animals.

\section{Group 3.-Shorter-fibre asbestos $(2.5 \mu)$ (7 animals).}

The use of a shorter-fibre asbestos results in a rather different tissue reaction. The lesions in these experiments are more scattered and affect the interstitial tissue and the alveolar walls more widely than in the previous experiment. This appears to be due to the fact that the process of phagocytosis is easier with the shorter than with the longer fibre. These fibres can be detected in the alveolar walls and in the perivascular and peribronchial lymphatics, and there is considerable hyperplasia of the peribronchial lymph nodes, which is absent in the longfibre experiments (Fig. 9). There are still, however, some nodular areas of foreignbody giant-cell reaction, particularly in the animals which survived for a shorter time. In these animals the increase in reticulin is mainly in the alveolar walls. 
The general change, therefore, is a fairly widespread thickening of the alveolar wall and some emphysema (Fig. 10). Brown pigment occurring in most of the experimental animals is mainly phagocytosed, and lies in the lymphatic channels and glands. Black pigment occurred in only minimal quantities, and that in the longest surviving animals.

\section{Group 4.-2,5 $\mu$ Asbestos plus aluminium (9 animals).}

The changes seen in the lungs of these rabbits are an intensification of those seen in the last experiment. The foreign-body reaction is more widespread and continues for a longer period. The lesions are interstitial as well as nodular. The increase in reticulin is chiefly confined to the alveolar walls, and black pigment is present only in the animals surviving longest.

\section{Discussion}

These experiments suggest that when asbestos is retained in the lung it will excite a connective-tissue response ultimately leading to fibrosis. The localization of the lesion depends, however, on the size of the asbestos fibre. In the case of a long fibre, which is too big for removal by phagocytosis and is not expectorated, the reaction is an intra-alveolar deposition of connective tissue. This results in a nodular distribution of fibrous tissue. With shorter fibres

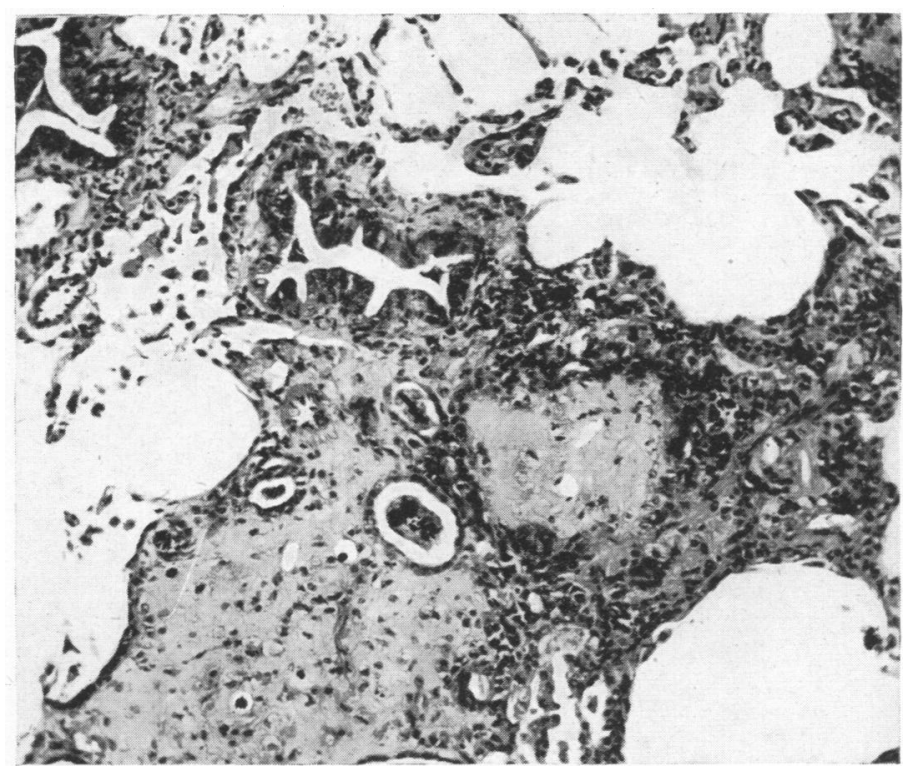

Fig. 11.-Long-fibre asbestos, showing asbestos body in bronchiole $(\times 112)$. 
capable of being mobilized into the alveolar walls and lymphatics the fibrous tissue is laid down in the alveolar walls, which ultimately leads to diffuse interstitial fibrosis. It seems possible that if asbestos fibres shorter than $2.5 \mu$ were used they would be completely removed from the alveolar walls and fail to produce an interstitial fibrosis.

The addition of metallic aluminium appears to be entirely without benefit, and serves merely to enhance and prolong the foreign-body reaction. The degree of fibrosis was certainly as great in all the experimental animals that had aluminium in addition to asbestos.

The brown pigment gives a positive reaction for iron and is presumably a haemoglobin derivative. The black pigment in some cases appears to surround asbestos fibres, and is presumably comparable with the familiar asbestos body in human asbestosis (Fig. 11).

Our results differ from those of Gardner in that asbestos fibres as short as $2.5 \%$ have produced an interstitial fibrosis. This may be due to the fact that we have been administering a known weight of asbestos (and aluminium) directly into the trachea. Gardner's animals were merely kept in an atmosphere laden with particles of asbestos. It is thus possible that the amount of asbestos fibres actually reaching the lung was very much smaller in amount in the case of Gardner's animals than in our own.

\section{SUMMARY}

1. Experiments on four groups of animals are described. The first was treated with $100 \mathrm{mg}$. of Rhodesian asbestos fibres $15 \mu$ long, intratracheally at monthly intervals. The second group was treated similarly, except that $2 \mathrm{mg}$. of metallic aluminium were added to each dose. In Group 3 the rabbits were treated as in the first group except that the asbestos fibres were only $2.5 \mu$ long. Group 4 had the same doses of asbestos as Group 3, but $2 \mathrm{mg}$, of aluminium was added to each dose.

2. All groups showed a foreign-body reaction in the lungs.

3. Animals in the long-fibre experiments developed a nodular reticulinosis comparable with the experimental silicotic nodule.

4. Animals in the short-fibre experiment developed a diffuse interstitial reticulinosis.

5. The addition of aluminium did not afford any benefit in any group.

\section{ACKNOWLEDGMENT}

Our thanks are due to Messrs. Turner Bros. Asbestos Co. for grant-in-aid to cover the cost of this investigation. To Dr. Bateman, Mr. Dawson, Dr. 
Merewether, Dr. Middleton, and Mr. Soothill, members of a Committee for the Study of Asbestosis, we extend our gratitude for much helpful advice and consultation. We thank Mrs. M. Gilchrist and Messrs. A. F. Hoffler, W. Weedon, and D. Bull for their technical assistance. The photographs were prepared by Mr. E. V. Willmott, to whom our thanks are also due.

\section{REFERENCES}

Belt, T. H., and King, E. J. (1945). Spec. Rep. Ser. M.R.C., London, No. 250.

Crombie, D. W., Blaisdell, J. L., and MacPherson, G. (1944). Canad. med. Ass. J., 50, 318 Gardner, L. U. (1937). First Progress Report on Asbestosis Experiments at the Saranac Lab. (Private Communication), May, 1937. i938.

(1938). The Second Progress Report on Asbestosis Experiments, April, (1938). Progress Report on Asbestosis Experiments (Private Communication), December, 1938.

(1940). Interim Report on Experimental Asbestosis at the Saranac Lab., 1940 (Private Communication).

(1942). Amer. Rev. Tuberc., 45, 762. 